\title{
Role of sympathetic nervous system in non-ischaemic ventricular arrhythmias
} \author{
PHILIPPE COUMEL, MICHAEL D ROSENGARTEN, JEAN-FRANCOIS LECLERCQ,
PATRICK ATTUEL \\ From Hôpital Lariboisière, Paris, France
}

SUMMARY Propranolol and nadolol were used in two groups of patients having ventricular arrhythmias. The two groups were characterised by differences in sympathetic drive. The 10 nonadrenergic patients had idiopathic, monomorphic extrasystoles (isolated with fixed coupling or in pairs or salvoes) arising from the right ventricle or the septum. These extrasystoles were chronic and benign, with a slightly increased daytime frequency (day:night $=1.6$ ). They disappeared on exercise. The nine adrenergic patients had less frequent but more complex polymorphic ventricular extrasystoles, and rapid and irregular tachycardias which were resistant. They occurred predominantly during the day and were associated particularly with stress and exercise. They were either idiopathic, or coexisted with mitral valve prolapse (three cases) or hypertrophic subaortic stenosis (one case) in young patients (mean age, 32 years) who did not have coronary heart disease. Nadolol was more effective than propranolol in controlling the arrhythmia, heart rate, and variations in sinus rhythm in the adrenergic group, while the arrhythmia was not controlled in the non-adrenergic group. Using clinical variables, comparison of the frequency of extrasystoles by day and night, and assessment of the antiarrhythmic effect of beta-blockers, the role of the sympathetic tone in nonischaemic ventricular arrhythmias may be elucidated.

The role of the sympathetic nervous system in ventricular arrhythmias has been shown experimentally ${ }^{1-3}$ and suggested by clinical observations. ${ }^{4-6}$ It has been difficult to determine which clinical variables most effectively demonstrate the significance of its influence, and hence the value of treatment by betablockade. This study uses clinical, electrocardiographic, and therapeutic indices to evaluate the role of sympathetic drive in 19 patients with ventricular arrhythmias of non-ischaemic origin.

\section{Subjects and methods}

GROUPS OF PATIENTS (Table)

The 19 patients in this study had ventricular arrhythmias in the absence of coronary heart disease or previous myocardial infarction, and there was no cardiac, respiratory, or gastrointestinal contraindication to beta-blockade. They were allocated to one of the two groups on the basis of their tolerance of the

Accepted for publication 21 September 1981 arrhythmia and the precipitating factors. Patients who could indulge in simple activities without palpitation were assumed to be insensitive to catecholamines and allocated to the non-adrenergic group. Patients complaining of palpitation that occurred exclusively in association with effort or that was aggravated by emotion were classified as adrenergic.

The non-adrenergic group comprised six male and four female patients, with a mean age of 41 (range = 10 to 67). The arrhythmia was discovered either at routine examination (cases 1 to 6 ) or as a result of investigating "skipping of heart beat" at rest. Cardiovascular examination included electrocardiogram and echocardiogram, clinical, and radiological examination. The normal physical activity of these patients was undisturbed by their idiopathic ventricular extrasystoles. The four male and five female patients of the adrenergic group had a mean age of 32 (range = 12 to 55), and suffered far more striking arrhythmias. Cases 11 to 13 were children with syncope of effort resulting from rapid and irregular ventricular tachycardia. They have been reported. ${ }^{7}$ Two young women (cases 14 and 15) had similar histories, but 137 
Table Clinical, electrocardiographic, 24 hour monitoring, and exercise data for non-adrenergic (cases 1 to 10) and adrenergic (cases 11 to 19) patients during control period and on beta-blocking therapy

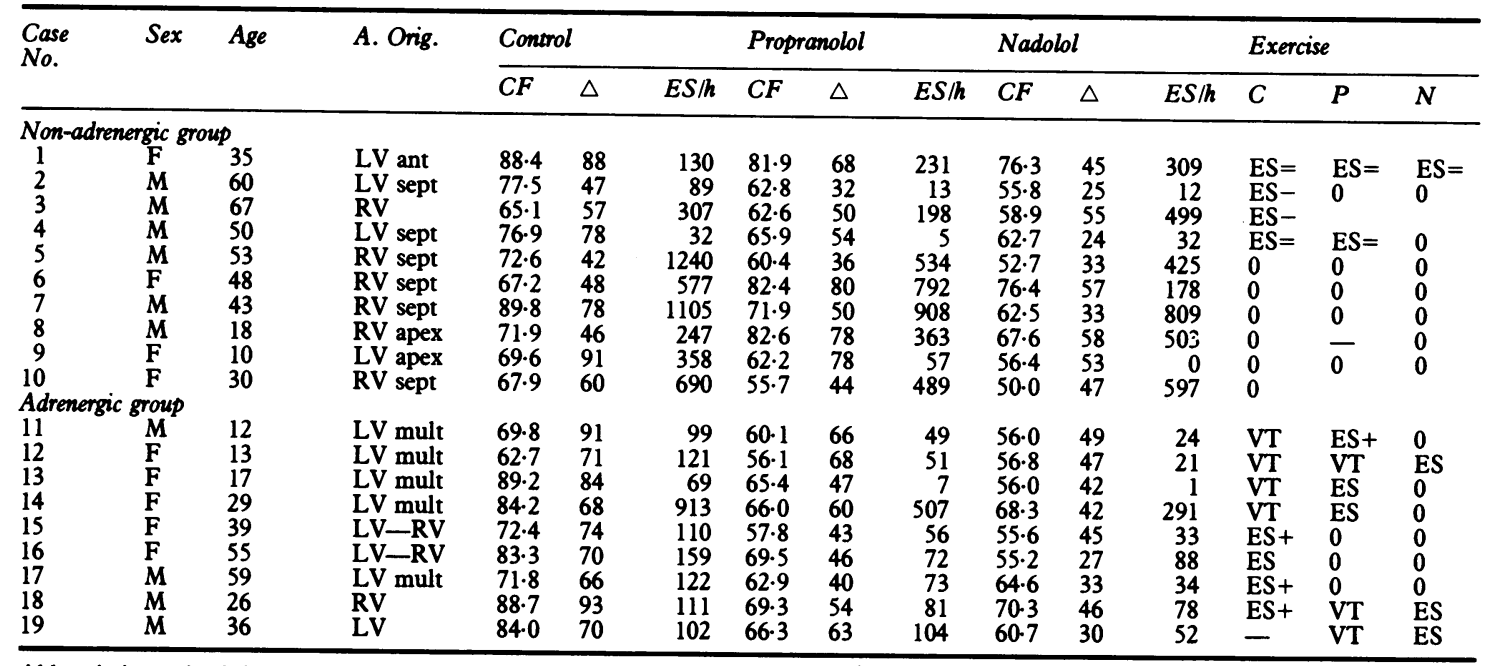

Abbreviations: A. Orig., arrhythmia origin; C, control; CF, cardiac frequency/minute; $\Delta$, range of sinus frequency in the 24 hour period; $\mathrm{ES} / \mathrm{h}$, hourly extrasystolic rate over the 24 hour period; $\mathrm{ES}=$, unchanged extrasystolic rate at exercise; ES-, decreased extrasystolic rate at exercise; ES + , increased extrasystolic rate at exercise; N, nadolol; P, propranolol; LV, left ventricle; RV, right ventricle; ant., anterior wall (of
the LV); mult, multiform; sept, septum.

(Note: cases 8 and 15 received acebutolol instead of propranolol.)

their symptoms were less severe and had evolved over a long period. Palpitation without dizziness was a feature of cases 16 and 17 . Finally there were two patients (cases 18 and 19) in whom exercise or emotional stress precipitated sustained paroxysmal ventricular tachycardia which was not self-limiting.

The adrenergic group underwent thorough investigation. All cases were subjected to cardiac catheterisation with left ventricular angiography. No risk factors were identified in this or the other group and there was no clinical or electrocardiographic evidence of coronary artery disease. Cases 18 and 19 underwent additional coronary angiography to exclude congenital abnormalities of the coronary arteries. Case 19 had undergone surgery four years previously for hypertrophic cardiomyopathy. Three cases of mitral valve prolapse were discovered (cases 14, 16, and 17). Urinary catecholamines were normal in this group, as were serum catecholamines in cases 11 to 13 , while the latter were at the upper limit of normal in case 19.

\section{ELECTROCARDIOGRAPHIC ANALYSIS}

Standard 12 lead electrocardiograms were obtained and the morphology of the ventricular arrhythmias was thereby demonstrated. Exercise tests were performed with electrocardiographic monitoring. Work increments of 20 to $30 \mathrm{~W}$ of one minute duration were used. The end-point in the non-adrenergic group was taken to be disappearance of the arrhythmia, while in the adrenergic group it was the onset of arrhythmia and symptoms. The same exercise test was repeated when the patient was on beta-blocking therapy.

Endocavitary studies were not routinely performed. In the non-adrenergic group, six cases were explored (cases $1,3,5,6,9,10)$. The remainder were not studied as their arrhythmias were considered too benign to justify invasive exploration and 24 hour monitoring yielded sufficient information. In the adrenergic group, all but one patient (case 15) were explored. Case 15 had an arrhythmia considered so severe that treatment was initiated. In all cases the arrhythmias were studied with atrial stimulation and with small doses of isoprenaline. In cases $9,10,18$, and 19, attempts were made to intitiate sustained ventricular tachycardia by right ventricular stimulation, but they failed.

\section{AMBULATORY ELECTROCARDIOGRAPHIC MONITORING}

The number of ambulatory recordings (using Avionics and Dynagram recorders) depended on clinical need and changes of medication. Previous electrocardiograms or 24 hour recordings were available for most patients while undergoing treatment other than that given during this study. They confirmed the stability of the arrhythmias. Patients in the non-adrenergic group were monitored for a mean of 6.8 days (range four to 13). Patients in the adrenergic group, with uncommon and more resistant arrhythmias, underwent monitoring for a mean of 12.3 days (range six to 22). A total of 17924 hour recordings was obtained: 49 control tracings (mean 
2.6, range one to six), 88 with nadolol (mean 4.6, range one to 19), and 42 with another beta-blocker (mean $2 \cdot 2$, range one to four).

The ambulatory recordings were analysed by the ATREC computer program. ${ }^{8}$ In addition to analysis of the 24 hour period, two periods, from 10.00 hours to 18.00 hours and 01.00 hour to 05.00 hours were analysed separately. The hourly rate for the following categories of extrasystole was computed: isolated ventricular extrasystoles, pairs, and salvoes. For salvoes, only the first 10 beats were taken to be ventricular extrasystoles, if followed by ventricular tachycardia. To simplify the results, the overall number of ventricular extrasystoles is reported. There were four cases with several control tracings of which those with runs of sustained ventricular tachycardia (in cases 18 and 19) or long salvoes (in cases 9 and 10) were eliminated. This was done as it was impossible to quantify the ventricular extrasystoles in these severe arrhythmias in a logical manner comparable to that for less severe arrhythmias. This bias did not lead to unmerited conclusions concerning the efficacy of treatment. It also gave a clearer quantitative breakdown for comparison with recordings while under treatment.

The mean heart rate was evaluated at 10 minute and hourly intervals. The overall cardiac rate was considered to be of sinus origin when the ventricular extrasystoles were isolated and followed by a compensatory pause. Frequent coupled beats, salvoes, sustained or frequent ventricular tachycardia result in apparently raised rates, but in these instances the ventricular rate does not reflect the sinus rate. In computing the minimum and maximum rates, by measurement of the fastest and slowest rates of a sliding 16 beat $\mathbf{R R}$ interval, these errors were eliminated by careful selection of the tracings used. The gradient "delta" of the sinus rate is determined by the difference between minimum and maximum rates over the 24 hour period and reflects the spontaneous variability of the sinus rate of ambulatory patients.

\section{TREATMENT WITH BETA-BLOCKERS}

Each patient underwent treatment with two betablockers, the order of which was randomised. Dosage of the drug was determined by weight and severity of the rhythm disturbance. In 17 patients, propranolol was used as the baseline medication, while acebutolol was used for two patients. Acebutolol, $200 \mathrm{mg}$, was taken to be equivalent to $40 \mathrm{mg}$ of propranolol. The q.i.d. dose of propranolol was $120 \pm 23 \mathrm{mg} /$ day for the non-adrenergic group and $142 \pm 60 \mathrm{mg} /$ day for the adrenergic group. Nadolol was administered once daily in the same dose as for propranolol. The minimum duration of treatment was four days for each drug, with a mean of seven days for propranolol and 12 days for nadolol. The difference is accounted for by the long-term use of nadolol in some adrenergic patients. Evaluation of each drug was performed after comparable durations of treatment.

Statistical evaluation was done on the raw data for the mean cardiac rate and the delta sinus rate, and after logarithmic transformation for the rate of ventricular extrasystoles. The Mann-Whitney test was used to compare the two groups and analysis of variance performed to compare the two therapeutic trials with the controls. Qualitative differences were checked by the exact test. Data are expressed as mean \pm standard deviation in the text, but by mean \pm standard error in the Figures.

\section{Results}

Indices including electrocardiograms, exercise tests, and ambulatory recordings were studied for the control and subsequent treatment periods. The two groups could not be differentiated on the basis of age $(p=0.25)$, sex $(p=0.29)$, or drug dosage $(p=0.80)$.

\section{BASELINE CHARACTERISTICS OF VENTRICULAR ARRHYTHMIAS}

(1) Origin and distribution of the arrhythmias (Table)

The QRS morphology is clearly different in the two groups of patients. In the non-adrenergic group, the origin of the ventricular extrasystoles is the right ventricle in six patients (one apical, four septal) and the left ventricle in four patients (two septal). All the complexes of ventricular origin are monomorphic (Fig. 1) except in case 3, where the first of the coupled beats was of right ventricular origin, while the second was either identical or arose elsewhere in the right ventricle.

In the adrenergic group, the origin was exclusively the left ventricle in six patients (cases $11,12,13,14$, 17,19 ), the right ventricle in one (case 18), and both right and left ventricle in two (cases 15 and 16) (Fig. 2). There were significant differences between the groups in terms of the ventricular origin of arrhythmias $(p<0.04)$ and in terms of the uniformity of the ventricular complexes $(p<0.001)$.

The coupling interval and the grouping of the arrhythmias also differentiate the two groups. In the non-adrenergic one, the coupling interval varied within a narrow range of 400 to $600 \mathrm{~ms}$, with the exception of case 1 which demonstrated true parasystole. The ventricular extrasystoles were isolated in cases $1,4,5$, and 6; pairs were present in cases $2,3,7$, and 8 , and there was frequent sustained ventricular tachycardia or repetitive long salvoes, almost regular at a rate of 150 to 200 per minute in cases 9 and 10 .

In the adrenergic group, a greatly changing coupling interval was the rule, with frequent $R$ upon $T$ phenomena. All patients had doublets and ventricular 


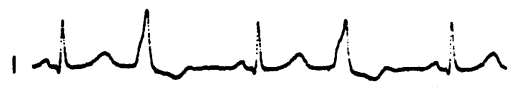

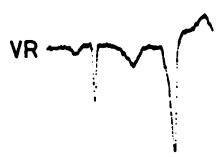

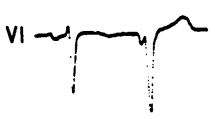

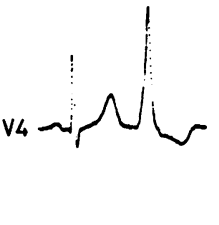

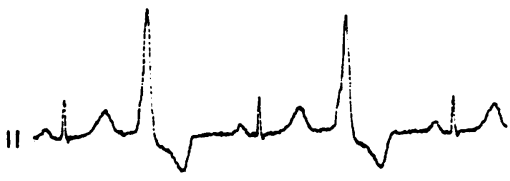<smiles>CCCC(C)C(C)CC</smiles><smiles>[Y2]C(I)CCC(C)CC</smiles>

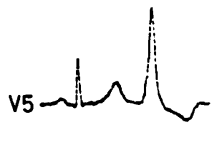

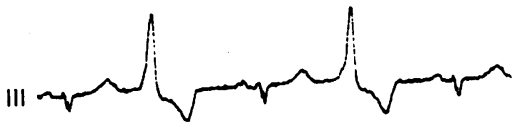

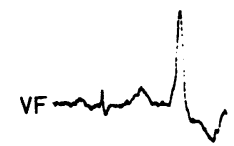

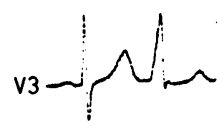

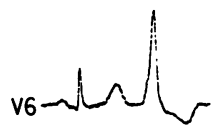

Fig. 1 Typical 12 lead electrocardiogram in the non-adrenergic group (case 6). $Q R S$ complexes of ventricular origin have a long and constant coupling interval, with frequent bigeminy. Note the vertical axis and the left bundle-branch block pattern, suggesting a high septal origin.
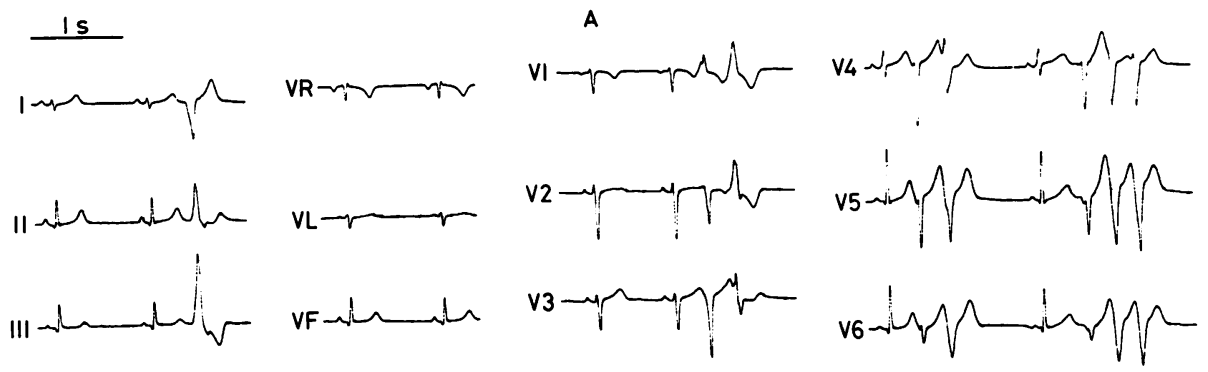

B

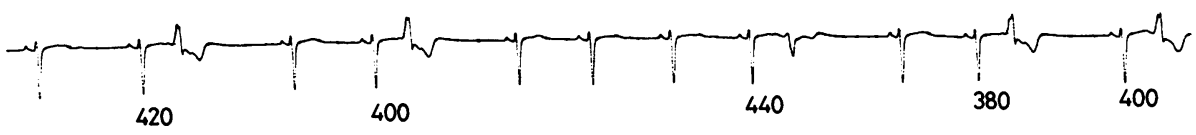

C

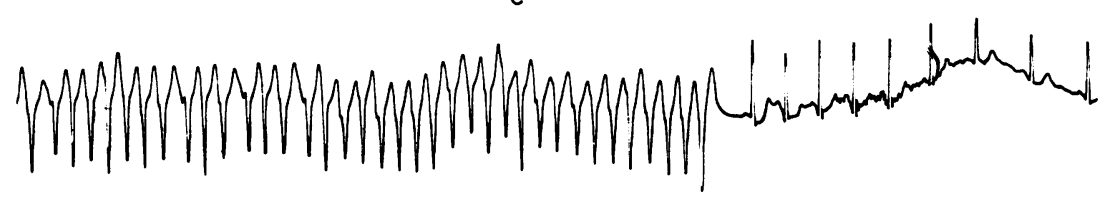

Fig. 2 Typical electrocardiogram in the adrenergic group (case 14). Ventricular extrasystoles are polymorphic, of left ventricular origin, and grouped in pairs or short salvoes $(A)$. The coupling interval is variable $(B)$. It is often short with $R$ upon $T$ phenomena. Runs of very rapid and irregular ventricular tachycardia are observed on exercise $(C)$.

tachycardia, the latter being monomorphic only in case 18. The ventricular tachycardia was of short duration (10 to 15 beats) in cases 16 and 17 , more sustained and irregular in cases 15 and 19 , and particularly rapid (up to $300 /$ minute), irregular, and polymorphic in cases 11 to 14 . The two groups showed a difference in the incidence of isolated ventricular extrasystoles and doublets as compared with salvoes and sustained ventricular tachycardia $(\mathbf{p}<0.001)$. 


\section{(2) Endocavitary electrophysiological tests}

Exploration was carried out in 14 patients and showed clearly different phenomena in the two groups, but did not establish the mechanisms of the arrhythmias. In four patients (cases 9,10,18, and 19), attempts at initiating or terminating ventricular tachycardia by pairs or repetitive stimuli did not give reliably reproducible results. The behaviour of the ventricular extrasystoles as a function of the atrial stimulation rate was variable. In the non-adrenergic group, five patients (cases $3,5,6,9,10$ ) had extrasystoles which were dependent on the atrial rate through a range of frequencies with minimum and maximum threshold values. This was not true of case 1 with parasystole. $\mathrm{Bi}-$, tri-, and quadrigeminy were seen to appear and disappear in relation to the atrial rate and occurred mostly with fixed coupling. These characteristics were disclosed on ambulatory monitoring in the remaining patients who were not explored. In the adrenergic group, the incidence of ventricular extrasystoles was hardly influenced by the atrial stimulation rate. They disappeared only by overdriving the ventricles and their frequency only increased on exercise testing, but not with acceleration of the pacing rate. The administration of small doses of isoprenaline gave results comparable with those obtained on exercise testing. In the non-adrenergic group, it reduced the number of ventricular extrasystoles precipitated by acceleration of the sinus rate. In cases 9 and 10 , however, it increased the duration and the rate of the salvoes while reducing their incidence. Of the patients in the adrenergic group who were explored and given isoprenaline, their ventricular arrhythmias were aggravated, with salvoes which were longer, faster, and poorly tolerated.

\section{(3) Exercise testing (Table)}

In six cases of the non-adrenergic group, the ventricular extrasystoles disappeared with effort on reaching a threshold value of the sinus rate (Fig. 3). In only two cases did the rate of ventricular extrasystoles diminish without complete disappearance. In a further two cases, the relative rate of ventricular extrasystoles was not modified by the sinus rate; hence there was an increase in the absolute number of ventricular extrasystoles per minute with the increase in the sinus rate.

All patients in the adrenergic group underwent

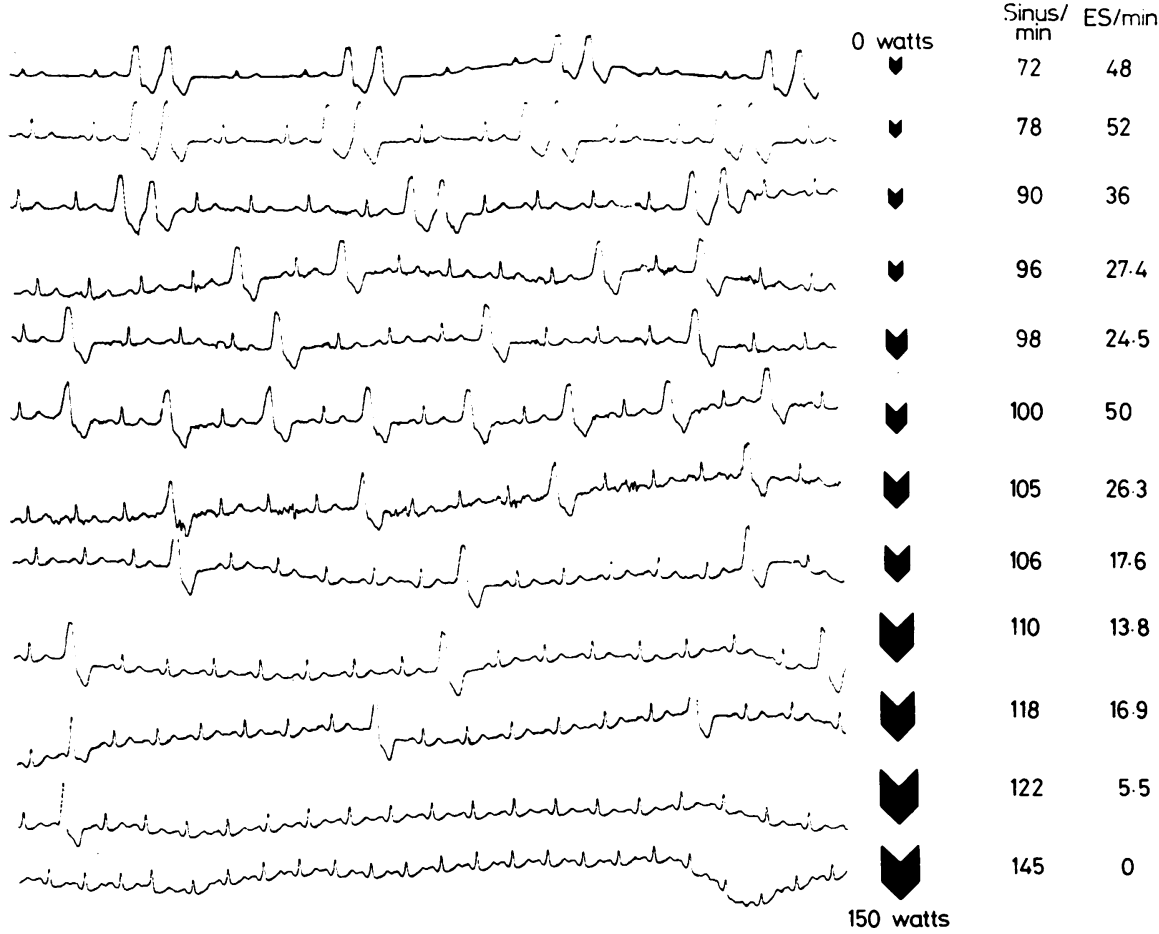

Fig. 3 Typical exercise profile of a non-adrenergic patient. As effort was progressively increased from 0 to 150 watts, the sinus rate increased from 72 to 145 beats/minute. The number of ventricular extrasystoles $(E S)$ is closely related to the sinus rate, viz. pairs, isolated ventricular extrasystoles, bigeminy, trigeminy, etc. The net result is either an increase or decrease in the absolute or relative ventricular extrasystolic rate. The general tendency, however, is a progressive disappearance of the ventricular arrhythmia. 

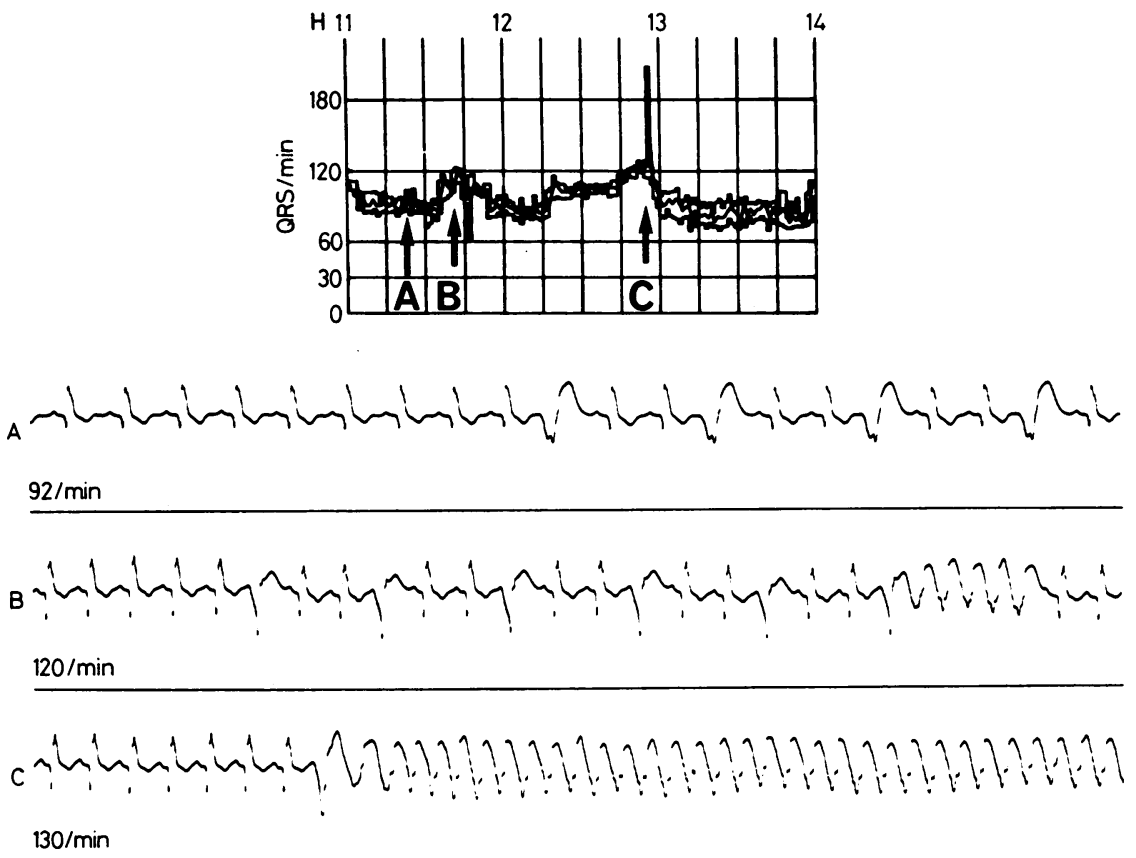

Fig. 4 Parallelism of sinus rate and ventricular arrhythmia in an adrenergic patient (case 19). Above is the computerised analysis of a three-hour continuous tracing expressed in terms of minimum, mean, and maximum frequency curves. The tracings corresponding to periods $A, B, C$ are shown below. During $A$, the sinus rate is $92 /$ minute and trigeminy is noted. During $B$, there is a rate of $120 /$ minute, the coupling interval of isolated ventricular extrasystoles is shortened, and a run of five beats of different morphology is seen. During $C$, the rate is $130 /$ minute, and ventricular tachycardia at a rate of 230 /minute occurs.

exercise testing with the exception of case 19 in whom exercise induced a poorly tolerated ventricular tachycardia (Fig. 4). In the remaining eight, exercise induced ventricular tachycardia in four, caused an increased frequency of short runs of ventricular tachycardia in three, and caused an absolute and relative increase in the frequency of ventricular extrasystoles in one.

\section{(4) Ambulatory electrocardiographic monitoring (Table,} Fig. 5)

In the non-adrenergic group, the hourly rate of ventricular extrasystoles was $478 \pm 439$. The difference between daytime $(591 \pm 462)$ and night time (361 \pm 514) rates was not significant ( $p>0.1$ ) with a ratio of 1.6. The mean cardiac rate over 24 hours was $74.7 \pm$ $8.6 / \mathrm{min}$ and was greater during the day $(82.9 \pm 9.6)$ than at night $(65.8 \pm 9.6)(\mathrm{p}<0.01)$, the ratio being 1.26. Delta over 24 hours was $64 \pm 20 / \mathrm{min}$, the average maximum rate was $121 / \mathrm{min}$, and the average minimum rate was $57 / \mathrm{min}$.

In the adrenergic group the hourly rate of ventricular extrasystoles was $201 \pm 267$. The difference between the daytime rate $(252 \pm 390)$ and the night time $(84 \pm 78)$ was significant $(p<0.05)$, with a ratio of 3.
The cardiac rate over 24 hours was $78.5 \pm 9.3$ and was greater by day $(87.0 \pm 10.5)$ than by night $(64.4 \pm$ $8.4)(p<0.01)$ with a ratio of 1.35 . Delta over 24 hours was $76 \pm 10$ with an average maximum of $130 / \mathrm{min}$ and average minimum of $54 / \mathrm{min}$.

There were significant differences between the two groups for the day:night ratios of rates of ventricular extrasystoles, 1.6 to $3(p<0.05)$ and heart rates, 1.26 to $1.35(p<0.05)$. There was a difference in the delta values $(p<0.05)$.

EFFECTS OF TREATMENT WITH BETA-BLOCKERS (Table, Fig. 6 and 7).

\section{(1) Effects of propranolol}

In the non-adrenergic group, propranolol (and 0 acebutolol when it was used) does not modify the 0 incidence of ventricular extrasystoles found on exercise testing $(\mathrm{p}=0.37)$ (Table). There is a decrease of $\stackrel{?}{+}$ $23 \%$ in the rate of ventricular extrasystoles in 24 hours, but this was not found to be statistically significant. Neither the average heart rate in 24 hours $(-8 \%)$ nor the delta value $(-11 \%)$ was significantly altered (Fig. 7). 

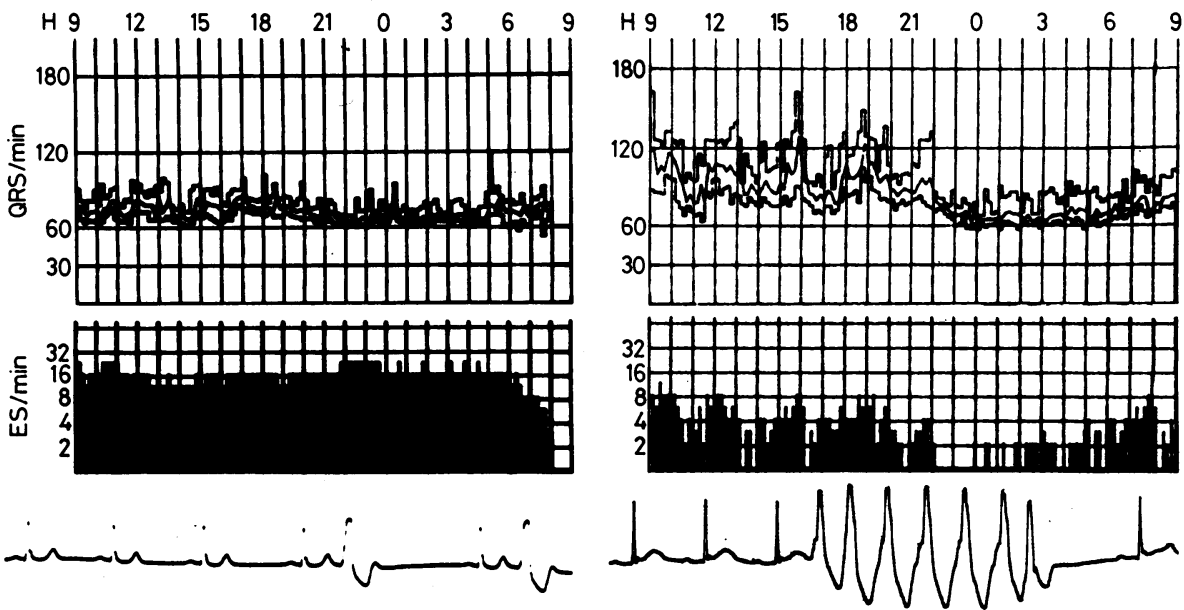

Fig. 5 Typical continuous 24 hour electrocardiogram for the non-adrenergic group (case 5) and for the adrenergic group (case 18). Left, the ventricular extrasystolic rate remains constant all day and the heart rate does not vary greatly between day and night. The minimum and maximum frequency curves closely approximate the mean heart rate, the difference, delta, being 40/minute. Right, the ventricular extrasystolic rate varies with the mean heart rate. Delta is greater, 70/minute.

In the adrenergic group, exercise testing during treatment with propranolol resulted in ventricular tachycardia in two cases as compared with four during the control period. Case 19, who did not undergo this test, was found to have persistent ventricular tachycardia, but at a reduced rate. Numerous ventricular extrasystoles were seen in three cases, yet none could be induced by exercise in a further three. There was an overall reduction in the number of ventricular extrasystoles in 24 hours of $46 \%(p<0.01)$. It is important to note that the reduction is significant only for the daytime incidence (Fig. 6). The cardiac rate fell by $19 \%$ over 24 hours $(\mathrm{p}<0.01)$ and delta by $29 \%(\mathrm{p}<0.01)$.

\section{(2) Effects of nadolol}

In the non-adrenergic group, exercise testing resulted in the almost complete disappearance of the ventricular extrasystoles. The number of extrasystoles over 24 hours was not modified ( $-6 \%)$ and there was no difference between daytime and night time incidence. The cardiac rate over 24 hours was reduced by $17 \%$ $(p<0.01)$ and the delta by $32 \%(p<0.01)$.

In the adrenergic group, there was no case of exercise-induced ventricular tachycardia, while ventricular extrasystoles were noted in only three patients. The number of ventricular extrasystoles over 24 hours was diminished by $66 \%(p<0.01)$ and was lower both by day and by night. The mean cardiac rate was reduced by $17 \%(p<0.01)$ and delta by $34 \%$ $(\mathrm{p}<0.01)$.

\section{Discussion}

From experimental and clinical observations, there is no doubt of the influence of sympathetic tone on ventricular arrhythmias, particularly in the presence of myocardial ischaemia.9-11 It remains difficult to establish, in individual cases, whether a particular arrhythmia is dependent on sympathetic drive. It seems that sympathetic tone plays either a major or a contributory role in the generation of arrhythmias. This is certainly true of ischaemic arrhythmias which depend on the vago-sympathetic balance, but has also been observed in denervated hearts. ${ }^{12}$ We therefore elected to eliminate patients with coronary artery disease from this study. The heart was normal in all respects, except for the arrhythmia, both at rest and on exercise in the 10 non-adrenergic patients. Coronary artery disease in the adrenergic group was excluded on the basis of the following: age (mean = 32 years), absence of history, symptoms, suggestive abnormalities on the electrocardiogram (both at rest and on exercise), left ventricular angiography and, in two cases, coronary angiography.

We deliberately selected patients with palpitation occurring only at rest or only on exercise, this being apparent from the history. It appears that this preliminary and simple qualitative selection screen correlates well with the more sophisticated and quantifiable tests subsequently performed. These included electrocardiogram, exercise tests, electrophysiology, 24 hour monitoring, and therapeutic findings. While 


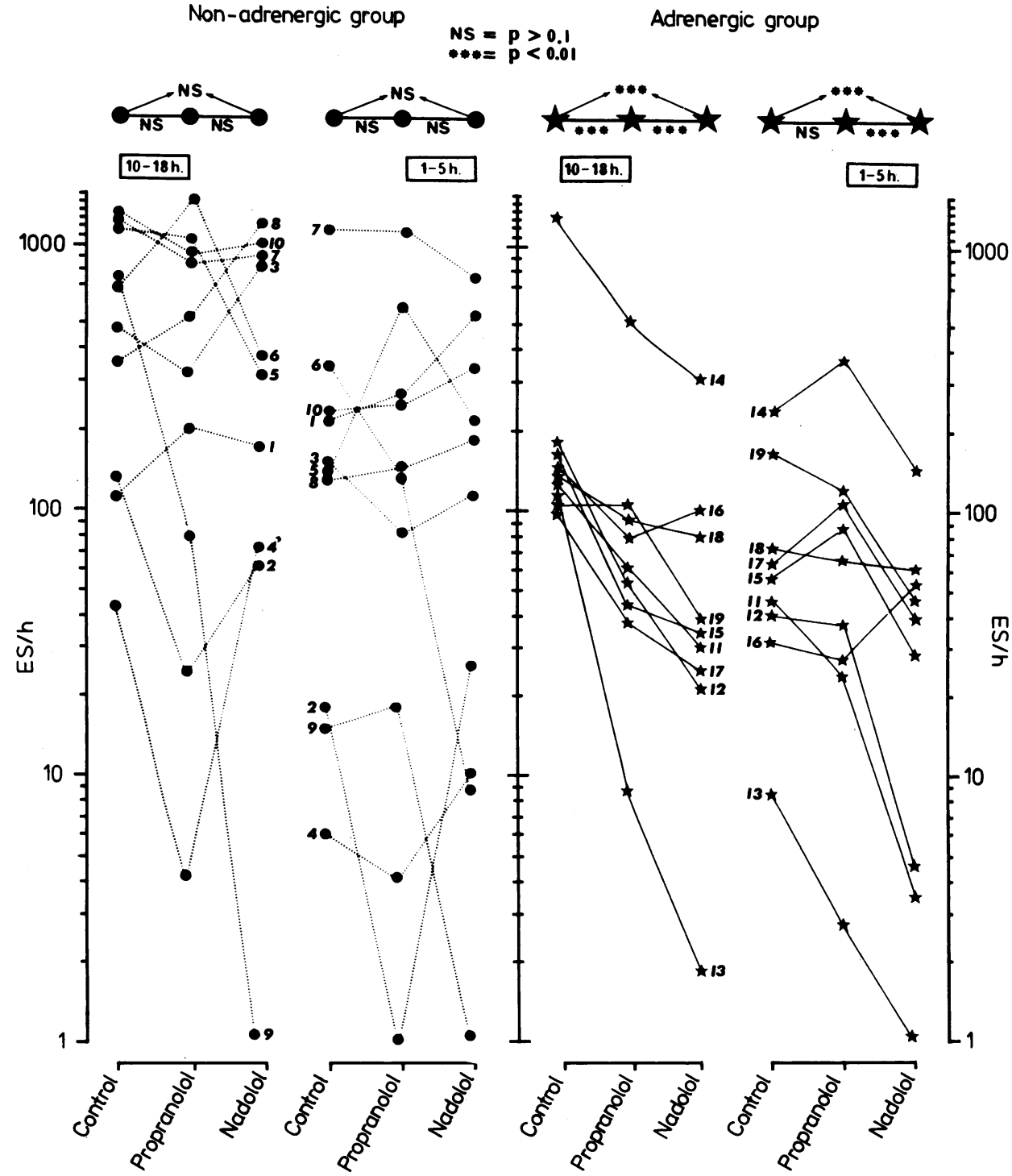

Fig. 6 Comparison of the hourly ventricular extrasystolic rate between the non-adrenergic group and the adrenergic, for both day and night and during control and therapeutic periods. Left, the non-adrenergic patients (solid circles). The left-hand column represents daytime, from $10 \mathrm{am}$ to $6 \mathrm{pm}$, and the right, night time, from 1 am to 5 am. There is no significant difference between the groups. Right, the adrenergic patients (solid stars). Left-hand column again shows daytime results, conversely the right-hand column. The daytime ventricular extrasystolic rate is significantly lowered by beta-blockade, especially by nadolol. At night, the effect is not significant with propranolol, but is significant with nadolol.

some indices were applicable to either group as a whole, there were discrepancies when applied to certain individual cases. For example, cases 9 and 10 of the non-adrenergic group shared characteristics with patients of the adrenergic group. The converse was true of cases 18 and 19 of the adrenergic group. None the less, two contrasting patterns of ventricular arrhythmia emerge from the cases studied. Thus assessment of the mechanism of the arrhythmias is facilitated and therapeutic consequences are disclosed.

NON-ADRENERGIC VENTRICULAR ARRHYTHMIAS The non-adrenergic patients were found to have 

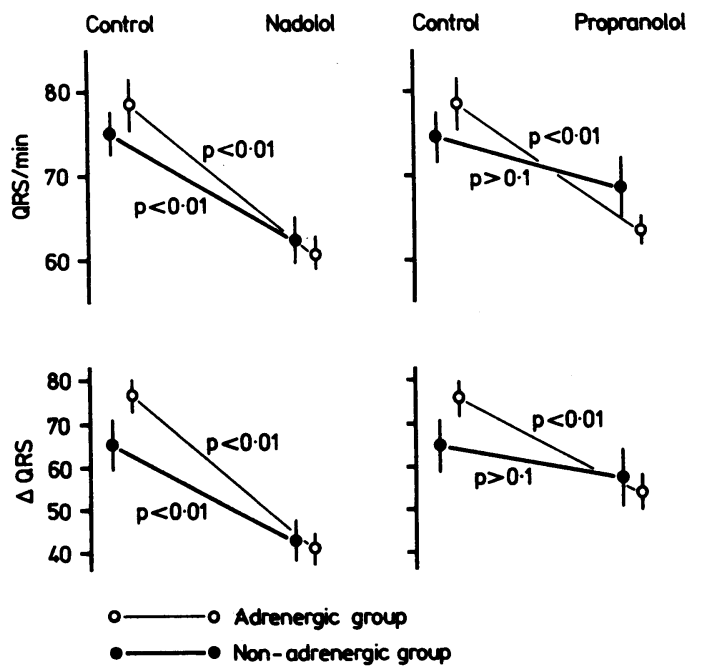

Fig. 7 Mean cardiac rate and delta values during control and treated periods. The mean cardiac rate over 24 hours is shown above. The control values (mean $+S E$ ) are not different for the two groups and both are significantly reduced by nadolol. Propranolol causes a significant reduction in the adrenergic patients only. Delta values are shown below and the same trends are seen. In addition, this index is significantly different for the two groups diring the control period $(p<0.05)$.

chronic and benign arrhythmias which were, in general, detected at routine examination. Some of these patients complained of a sensation of the heart skipping beats, occurring at rest and disappearing on exercise. There was no organic heart disease in this group. The ventricular extrasystoles were mostly monomorphic, being of right ventricular or septal origin, with a relatively long coupling interval which varied within narrow limits (400 to $600 \mathrm{~ms}$ ). Bi-, tri-, and quadrigeminy was frequently observed and the occurrence of arrhythmias was dependent on the sinus rate $^{1314}$ between two thresholds. Usually the lower one was apparent at rest, or at night, from 24 hour electrocardiograms. More important is to determine the upper one by accelerated atrial pacing or exertion. It was the arrhythmia observed during, and not after, exercise which was subject to evaluation. A range of arrhythmias was seen, from paired beats, which were not unusual, to sustained ventricular tachycardia. Salvoes three or 10 beats long, or even 20 , were quite frequent, with rates of 150 to $200 / \mathrm{min}$. When paired beats or short salvoes were seen, their rate and duration sometimes increased initially with effort before subsequently disappearing, as did the isolated ventricular extrasystoles.

\section{ADRENERGIC VENTRICULAR ARRHYTHMIAS}

These arrhythmias present a clear contrast to the pre- ceding group. They are far less common, the nine cases being drawn from a five year period during which patients were referred to our department specifically for management of severe arrhythmias refractory to treatment. Had the "control" group of non-adrenergic patients been collected over the same period, there would be in excess of 10 or 20 times the number in the group. The adrenergic arrhythmias were, in all cases, symptomatic and in certain instances, life-threatening.

Organic heart disease was established in four patients. The three cases of mitral valve prolapse supported the hypothesis of an associated hyperadrenergic state. ${ }^{15}$ Purely catecholamine-dependent arrhythmias, with no detectable heart disease, were clearly seen in three children in this group. ${ }^{7}$ Raised levels of urinary catecholamines were not, however, found in the adrenergic group; thus the arrhythmias appear to be related to catecholamine sensitivity rather than to a hyperadrenergic state.

The arrhythmias were polymorphic, of left ventricular origin, and with a variable but often short coupling interval. There were short, rapid, and irregular salvoes, the rate of which often exceeded $200 / \mathrm{min}$. Exercise tests were limited by the symptoms induced in every case. Significant electrocardiographic abnormalities were noted before the patients, accustomed to their arrhythmias, started to complain of symptoms. Atrial pacing did not influence the arrhythmias, in contrast to both infusion of isoprenaline and exertion. The sensitivity to the isoprenaline, but not the dosage, differentiates the two groups studied.

\section{AMBULATORY MONITORING}

Ambulatory recordings showed that the rate of ventricular extrasystoles for the non-adrenergic group was higher than for the adrenergic one $(p<0.05)$. The Table and Fig. 6, however, indicate that this criterion cannot be used to differentiate the individuals from either group. The rate of ventricular extrasystoles varied within wide limits and was a function of activity. It decreased with activity in the non-adrenergic patients and increased with the others. The day:night ratio for the rate was found to be a more reliable expression of the same concept, again showing a significant difference between the two groups $(p<0.05)$. Subjects could not be differentiated on this basis either. It can be seen from Fig. 6 that only one adrenergic patient exceeds a nocturnal ventricular extrasystolic rate of 100 /hour, while six patients of the non-adrenergic group exceeded this rate. Because of a lower threshold sinus rate for arrhythmias, the remaining four non-adrenergic patients were found to have a low rate of ventricular extrasystoles during sleep. ${ }^{16}$ The mean cardiac rate was not significantly 
different in the two groups, though higher in the adrenergic group. The day:night ratio (1.26:1.35, non-adrenergic:adrenergic) and the delta values were, on the other hand, significantly different $(p<0.05)$. The latter values express the maximum amplitude of the variation in sinus rate and are very sensitive, reflecting the pattern given by the computer analysis (Fig. 5).

\section{THERAPEUTIC EFFECT OF BETA-BLOCKADE}

Two drugs were used, of which propranolol served as a reference and nadolol as the more powerful agent. This represents a further approach to the problem of the mechanism of arrhythmias. The role of other drugs as membrane stabilisers is not discussed in this article. The two groups respond in opposite ways to quinidine and quinidine-like agents, however, the non-adrenergic patients being sensitive and the adrenergic patients being resistant. The converse was true for beta-blockers.

The arrhythmias were not suppressed in the nonadrenergic group by propranolol or nadolol, though they may have been influenced. Fig. 6 clearly shows that the rate of ventricular extrasystoles may rise, fall, or remain the same with either drug by day or by night. These changes were mainly related to the sinus rate, the arrhythmias featuring in the range of sinus rates between upper and lower threshold values. Thus the arrhythmias may have been more or less manifest on this basis. In the adrenergic group, however, there was always improvement with beta-blockade. Complete control of the arrhythmias was not necessarily achieved though the arrhythmias were heavily dependent on sympathetic drive, and some patients improved further on addition of quinidine. Detailed examination of the results showed that in the nonadrenergic group, the rate was unchanged by nadolol ( $-6 \%$ ) but diminished by $23 \%$ by propranolol. This is not statistically significant, but raises the question of the quinidine-like effect of propranolol which has been proven experimentally yet is not supported by clinical evidence.

In the adrenergic group, nadolol is effective by day and night, whereas propranolol was effective only by day. This suggests that the dependence of the arrhythmias on the sympathetic drive is more obvious by day, so that the less powerful agent (propranolol) has a more visible effect during the day. Propranolol and nadolol have different effects on the cardiac rate and the delta value. These are unaffected by propranolol in the non-adrenergic group, but quite significantly altered in the adrenergic group. Nadolol, on the other hand, modifies these indices in both groups, though to a greater extent in the adrenergic group. While there is a difference in the control values for delta between the two groups, no such difference exists for cardiac rate or delta while nadolol was administered.

These quantifiable results correlate with the symptomatic improvement described by the patients, which was greater with nadolol than with propranolol. Currently, six patients (cases 11 to $13,15,18,19$ ) are controlled on long term nadolol therapy, having been incompletely controlled by propranolol alone. It may be argued that propranolol appears less effective, but that this is attributable to using the same doses for both nadolol and propranolol. If, however, the dose of the latter is doubled, only modest therapeutic gain is achieved. No such gain was noted when the dose of nadolol was doubled.

In conclusion, there appears to be correlation between ventricular extrasystoles, cardiac rate, and the maximum variations in sinus rate on the one hand, and clinical symptoms and electrocardiographic findings both at rest and on exercise. ${ }^{17}$ The behaviour of the arrhythmias observed under the influence of beta-blockade confirms that there is an important contribution from the sympathetic nervous system in certain ventricular arrhythmias. Conversely, the absence of antiarrhythmic effect noted in other cases treated with beta-blockers sheds light on the mechanism of these. It is also of value in establishing the indication for using various drugs. The cases intermediate between the extremes of the range studied may represent a mixed picture where an arrhythmia is influenced by, but is not completely dependent on, sympathetic drive, providing an indication for combined therapies.

\section{References}

1 Corr PB, Gillis RA. Autonomic neural influences on the dysrhythmias resulting from myocardial infarction. Circ Res 1978; 43: 1-9.

2 Randall WC, Euler DE, Jacobs HK, Wehrmacher W, Kaye MP, Hageman GR. Autonomic neural control of cardiac rhythm: the role of autonomic imbalance in the genesis of cardiac dysrhythmias. Cardiology 1976; 61: 20-36.

3 Verrier RL. Neural factors in ventricular electrical instability. In: Kulbertus HE, Wellens HJJ, eds. Sudden death. The Hague, Boston, and London: Martinus Nijhoff, 1980: 137-55.

4 Corday P, Dodge HT, eds. Symposium on identification and management of the candidate for sudden cardiac death. Am F Cardiol 1977; 39: 813-934.

5 Coumel P, Leclercq JF, Rosengarten MD, Attuel P, Milosevic D. Unusual forms of severe ventricular tachyarrhythmias: their relationships with the QT interval and the vago-sympathetic balance. In: Kulbertus HE, Wellens HJJ, eds. Sudden death. The Hague, Boston and London: Martinus Nijhoff, 1980: 199-215.

6 Schwartz PJ. The long QT syndrome. In: Kulbertus HE, Wellens HJJ, eds. Sudden death. The Hague, Bos- 
ton, and London: Martinus Nijhoff, 1980: 358-78.

7 Coumel P, Fidelle J, Lucet V, Attuel P, Bouvrain Y. Catecholamine-induced severe ventricular arrhythmias with Adams-Stokes syndrome in children: report of four cases. Br Heart F 1978; 40, suppl: 28-37.

8 Attuel P, Rosengarten M, Leclercq JF, Milosevic D, Mugica J, Coumel P. Computer quantitated evaluation of cardiac arrhythmias. Pace 1981; 4: 23-35.

9 Frishman W. Clinical pharmacology of the new betaadrenergic blocking drugs. Part 9. Nadolol: a new longacting beta-adrenoceptor blocking drug. Am Heart $\mathcal{f}$ 1980; 99: 124-8.

10 Bergamaschi $M$. Role of the sympathetic and parasympathetic innervation in the genesis of ventricular arrhythmias during experimental myocardial ischemia. In: Schwartz PJ, Brown AM; Malliani A, Zanchetti A, eds. Neural mechanisms in cardiac arrhythmias. New York: Raven Press, 1978: 7-18.

11 Pantridge JF. Autonomic disturbances at the onset of acute myocardial infarction. In: Schwartz PJ, Brown AM, Malliani A, Zanchetti A, eds. Neural mechanisms in cardiac arrhythmias. New York: Raven Press, 1978: 7-10.

12 Mason JW, Stinson EB, Harrison DC. Autonomic nervous system and arrhythmias: studies in the transplanted denervated human heart. Cardiology, 1976; 61: 75-87.
13 Jalife J, Moe GK. Effect of heart rate on ventricular parasystolic activity. In: Schwartz PJ, Brown AM, Malliani A, Zanchetti A, eds. Neural mechanisms in cardiac arrhythmias. New York: Raven Press, 1978: 19-28.

14 Coumel P, Leclercq JF, Attuel P, et al. Tachycardies ventriculaires en salves. Etude électrophysiologique et thérapeutique. Arch Mal Coeur 1980; 73: 153-64.

15 Boudoulas H, Wooley CF, Reynolds J, Mazzaferri E. Mitral valve prolapse syndrome. Evidence for a hyperadrenergic state (abstract). Am $\mathcal{F}$ Cardiol 1979; 43: 368.

16 Lown B, Tykocinski M, Garfein A, Brooks P. Sleep and ventricular premature beats. Circulation 1973; 48: 691701.

17 Sonnhag C, Nylander E. Significance and reproducibility of exercise-related idiopathic ventricular arrhythmias. Eur Heart f 1980; 1: 183-93.

Requests for reprints to Dr P Coumel, Hôpital Lariboisière, 2 Rue Ambroise-Paré, 75475 - Paris Cedex 10, France. 